\title{
OBSERVAÇÕES SÔBRE A FAUNA BÊNTICA DO COMPLEXO ESTUARINO-LAGUNAR DE CANANÉIA (SP)
}

\author{
(Recebido em 3/3/1970)
}

\author{
Luiz RoBerto TOMmasi
}

Instituto Oceanográfico da Universidade de São Paulo

\section{SYNOPSIS}

The benthic fauna of the muddy bottoms of the estuarine lagunar complex of Cananéia (SP) is poor. It is dominated by the polychaets species. The gastropod Littoridina australis var. nana occurs in high density in the northern region of the Cananéia island. Some species, occurs only in the Trapandé bay, near of the Cananéia bar. Five faunistic zones was possible recognize on the region, i.e., zone of Renilla reniformis, Virgularia sp. and ophiuroids, zone of Macoma sp. and Tellina alternata, zone of Kalliapseudes schubartii and Phoronis sp., zone of Littorina australis var. nana and zone of hard bottoms. The Trapandé bay presents the richest muddy infralittoral benthic fauna of the complex. The influences of rivers and "marigots" in this fauna is pronounced.

The fauna of the infralittoral stone bottom, of the Casqueiro Island is described. A hypothesis is preserte explaining the existence of that fauna.

\section{INTRODUÇÃO}

O complexo estuarino-lagunar de Cananéia (apud Teixeira, 1969), situado no sul do Estado de São Paulo, (Fig. 1) tem sido estudado por diversos pesquisadores (Teixeira \& Kutner, 1961; Teixeira, Tundisi \& Kutner, 1965; Kutner, 1962; Magliocca \& Kutner, 1964). Trata-se de região muito complexa devido às mudanças periódicas que se verificam. Devido principalmente às correntes de maré, ocorre nessa região um complexo de misturas devido ao encontro das águas do mar, com as da região interior. Por êsse motivo, a sua macrofauna bêntica é pobre em relação a das zonas oceânicas vizinhas. Êsse fato está de acôrdo com as observações de Hesse et al. (1937), de que "sob a pressão de condições que demandam adaptação, o número de espécies diminui". À medida que penetramos na região lagunar, o ambiente bentônico assume, em função de sua hidrologia e tipos de sedimento, as características de "pessimus" (ver Allee et al. 1963). O mesmo foi por nós observado anteriormente (TоммAsı, 1967) para as partes mais internas da região estuarina de Santos (SP).

No presente trabalho, apresentamos algumas observações sôbre a fauna bêntica infralitoral, do complexo estuarino-lagunar de Cananéia (SP).

Publ. n.॰ 298 do Inst. Ocean, da USP.

\section{OBSERVAÇÕES SÔBRE A REGIÃO ESTUDADA}

a) Propagação da onda de maré e direção das correntes

Segundo as observações de Miniussi (1959), a onda de maré vinda do oceano após passar pela barra de Cananéia, divide-se em dois ramos, um para o Mar Pequeno e outro para o Mar de Cubatão, através da Baía de Trapandé. No local denominado Rio Guapara, as duas ondas se encontram, ocorrendo aí o fenômeno do "tombo de água". Na maré vazante, as águas ocorrem em direção contrária, fluindo para a barra de Cananéia, através do Mar do Cubatão - Mar Itapitangui - Baía de Trapandé e do Mar de Cananéia.

\section{b) Plâncton e Hidrografia}

Teixeira, Tundisi e Kutner (1965), verificaram que o fitoplâncton da região lagunar de Cananéia é constituído primàriamente de diatomáceas e é muito mais abundante do que o zooplâncton em número de organismos. O gênero do fitoplâncton mais comum foi a diatomácea Skeletonema sp.. Flagelados ocorreram em abundância, principalmente em abril (1962) na região onde se encontram os mares de Cananéia e de Cubatão. Os copépodos são os principais elemen- 


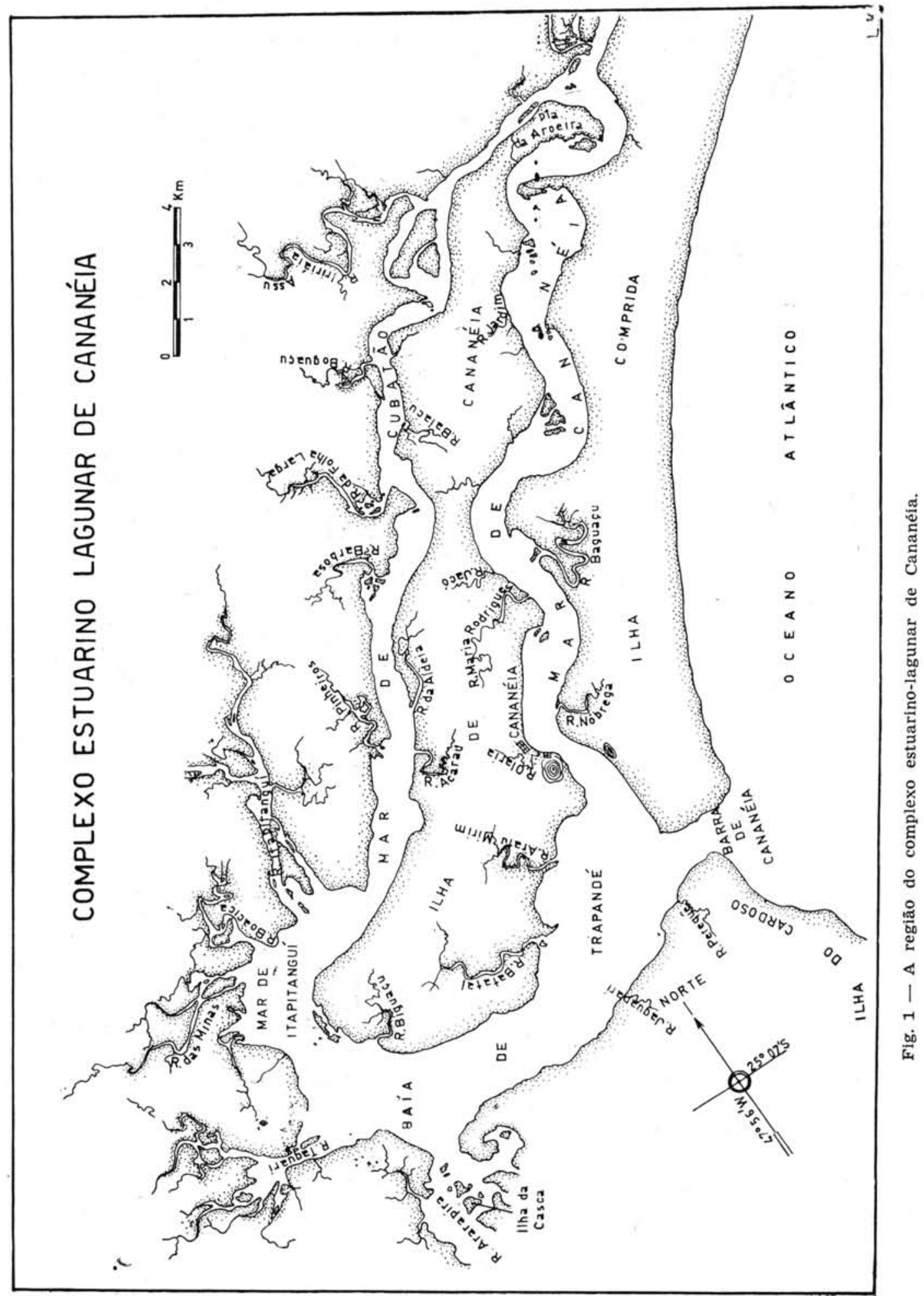


tos do zooplâncton, constituindo mesmo, sua grande maioria. Há uma predominância de herbívoros, sôbre os carnívoros nesse zooplâncton. Verificaram também a existência de uma grande turbidêz da água, bem como uma grande quantidade de "substância amarela" dissolvida na mesma. Êsses dois fatôres determinam uma acentuada redução da penetração da luz e, portanto, da zona eufótica em tôda a região. Verificaram haver um aumento gradativo do "standingstock" do plâncton, da barra para o interior da região.

Esse "standing-stock" apresenta uma correlação inversa com a clorinidade. Nas regióes próximas de desembocaduras de rios, há uma mistura de água doce com água salgada o que determina a existência de condições favoráveis a um rápido crescimento da população de fitoplâncton e conseqüentemente de zooplâncton. Verificaram também que as correntes de maré influenciam a distribuição do plâncton e promovem uma mis. tura de águas oceânicas com águas interiores, determinando assim, variação na salinidade, conteúdo em oxigênio e em outras condições da água da região. Durante a maré baixa, há carreamento de nutrientes e de plâncton da região lagunar para fora da mesma (TEIXeIra, Tundisi \& KutNer, op. cit.).

Teixeira, Tundisi \& Kutner (1965) e Kato (1966) mostraram o alto teor de oxigênio dissolvido na água de superfície e de fundo e a freqüente ocorrência, na entrada da região lagunar, de água com salinidade de 30\% . Segundo Tundisi \& Tundisi (1968) a magnitude das modificações de salinidade durante um período de 6 horas a $5 \mathrm{~m}$ de profundidade foi menor na região da barra de Cananéia do que em regióes mais internas como no Argolão e no Rio Baguassu.

\section{c) Fauna bêntica}

Muito pouco se conhece sôbre a fauna bêntica do complexo estuarino-lagunar de Cananéia. Os únicos trabalhos que apresentam muitas observações sôbre êsse ambiente e a sua flora e fauna são os de GERLACH $(1958 ; 1963)$.

\section{d) Sedimento}

Segundo Kutner (1962) a granulometria dos sedimentos está em desacôrdo com as características de região lagunar. MAGLiocCA \& Kutner (1964) estudando o conteúdo orgânico dos sedimentos da região de Cananéia, verificaram que apenas a região compreendida pela barra de Cananéia, Baía de Trapandé, Mar Itapitangui e parte de Cubatão, apresenta condições de deposição de região lagunar, isto é, uma gradação de valôres baixos e valôres altos de matéria orgânica (de ambiente marinho e terrígeno). Por êsses motivos, creio que é melhor o conceito de Teixeira (1969), de que se trata de um complexo estuarino-lagunar. Segundo KUTNER (op. cit.), a complexidade da região é devida a vários fatôres entre os quais: a ação conjunta da conformação fisiológica e do mecanismo de propagação de maré; a influência exercida sôbre a sedimentação por parte das correntes de maré enchente que se revelam mais competentes do que as de maré vazantes; a deposição no canal principal de grande quantidade de material silte-argiloso por parte dos inúmeros "marigots" que aí desaguam e por fim, as anomalias locais conferidas por feições topográficas particulares do fundo (poços, baixíos, etc.). É importante acentuar a observação dêsse autor sôbre a influência de rios, verdadeiros ou não, no sedimento da região. Todos os trechos do canal que recebem águas insulares ou continentais são bem caracterizados quanto à natureza do fundo, pois ali passam a dominar porcentualmente as frações silte-argilosas, havendo, conseqüentemente, uma diminuição sensível do diâmetro médio das partículas e perda de seleção granulométrica. As águas salgadas ao penetrarem pelos rios adentro, floculam as argilas em suspensão, determinando um depósito de caráter argiloso nos leitos dêsses rios. Na baixamar ou em épocas chuvosas, a corrente fluvial tem sua com. petência aumentada o que causa erosão e transporte de maior quantidade de material fino para as desem. bocaduras (KUTNER, op. cit.).

Os sedimentos de fundo da região de Cananéia são, de um modo geral, granulomètricamente homogêneos. São constituídos principalmente de areias finas e muito finas; as dimensões dos grãos médios (medianas) estão compreendidas entre 100-150 micra, sendo êsses sedimentos geralmente muito bem ou bem selecionados.

Magliocca \& Kutner (op. cit.) verificaram que a zona fronteiriça à entrada da barra, bem como todo o Mar de Cananéia é a região de menor concentração de matéria orgânica de tôda a região. Os valôres da relação $\mathrm{C} / \mathrm{N}$ indicam condições para uma decomposição mais ativa de matéria orgânica. Esta área está sujeita diretamente à ação de águas marinhas e não conta com a presença de rios de grande porte. A área que compreende o Mar de Itapitangui e parte do Mar de Cubatão é a mais rica em matéria orgânica, isso devido à presença de enorme contingente de águas fluviais e à pequena influência de águas marinhas. Os valôres de $\mathrm{C} / \mathrm{N}$ indicam tratar-se de uma área, provàvelmente, sob condições mais redutoras. As áreas situadas entre essas duas, anteriormente citadas, recebem influência tanto de águas marinhas como de águas fluviais e apresentam teor de matéria orgânica compreendida entre os valôres observados para aquelas áreas. Esses autores, analisando amostras obtidas nas margens da baía de Trapandé, verificaram que o sedimento dessas regiões apresenta baixo teor em matéria orgânica, o que mostra que a mesma não é depositada nas margens dessa baía, mas carreada para a mesma pelos rio que ai ocorrem.

\section{MATERIAL E MÉTODOS}

Em agôsto de 1964 foram realizadas 60 estações no complexo estuarino-lagunar de Cananéia (Fig. 2). Em cada uma dessas estações foram efetuados de 1 a 3 lances com um pegador do tipo Foerst "Petersen grab" modificado de $1 / 10 \mathrm{~m}^{2}$, obtidas amostras de sedimento, tomada a temperatura da água de fundo, bem como obtidas amostras da mesma para dosagem de salinidade. Os resultados dessas análises são apresentados na Tabela I. 


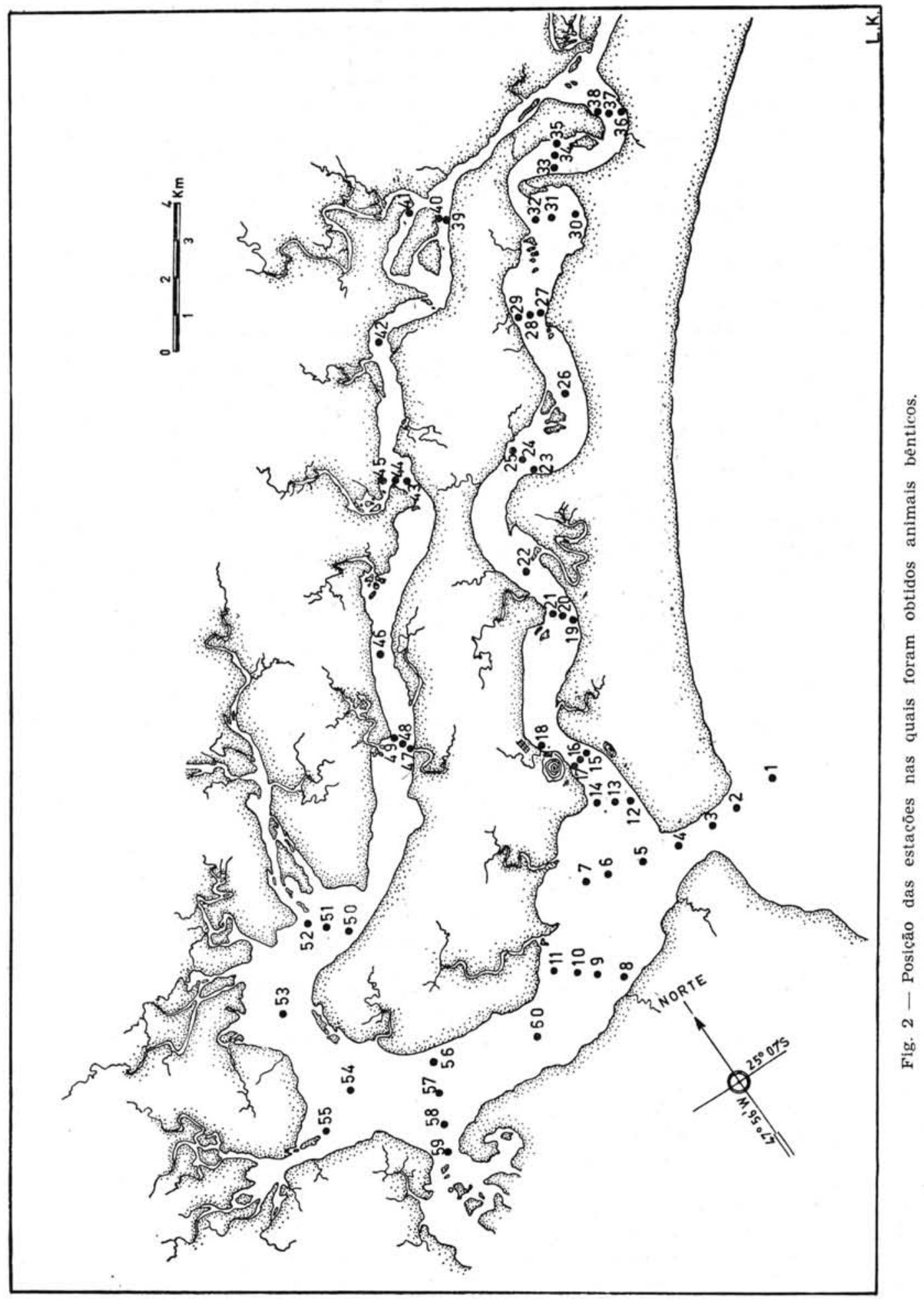


Dando continuidade aos trabalhos anteriormente citados, realizamos em fevereiro de 1967, 7 estações na região da Ilha da Casca situada na entrada do Rio Ararapira (Fig. 3), a qual não tinha sido por nós anteriormente amostrada.

As amostras bênticas foram lavadas e triadas em peneiras com malhas de 5,2 e $0,5 \mathrm{~mm}$. Os animais (macrofauna) foram imediatamente fixados em álcool a $70 \%$. Foram posteriormente separados por grupo, contados e pesados (pêso alcoólico). Visando preservar a integridade dos exemplares para estudos de taxonomia, foi tomado apenas o pêso alcoólico. Essa técnica danifica pouco o material, ao contrário do pêso sêco que provoca alterações irreversíveis.

Apesar das dificuldades em converter pêso alcoólico para pêso sêco (ver Thorson, 1957) e, portanto, calcular corretamente a biomassa bêntica, resolvemos apresentar os presentes dados, ainda que nesta forma, devido a: $10^{\circ}$ - o largo desconhecimento da fauna bêntica da região; $2 .^{\circ}$ - modificação que tem se operado nessa fauna com a eliminação de diversas espécies, provàvelmente como conseqüência de variação nas complexas condições hidrológicas existentes na região.

\section{A fauna bêntica do complexo estuarino-lagunar de Cananéia}

O número de indivíduos de cada grupo, por metro quadrado e o seu pêso alcoólico, foram condensados na Tabela I. Na Tabela II são apresentadas as espécies encontradas nas diversas estações.

Como se verifica dessas tabelas, a fauna bêntica da região lagunar de Cananéia é amplamente dominada pelos poliquetas. Dêsses animais, as espécies mais comuns, na baía do Trapandé foram Loandalia americana, Laonice japonica e maldanídeos como Clymene sp. e Clymenella sp.. Êsse grupo de poliquetas é o que ocorreu em maior número de estações, mas foj restrito à região compreendida entre a foz do Rio Ara. cau (no Mar de Cubatão) e a entrada do Mar de Cananéia. A região onde obtivemos uma maior densidade de poliquetas (mais de 40 indivíduos/1/10 $\mathrm{m}^{2}$ ) foi a situada entre a foz do Rio Aracau (Mar de Cubatão), Mar de Itapitangui e Baía do Trapandé até a região da foz do Rio Jaguapari, na mesma região onde os maldanídeos ocorreram.

Os lamelibrânquios não foram dominantes, em número de indivíduos, em nenhuma das estações realizadas. Das espécies obtidas devemos ressaltar Tellina alternata que ocorreu apenas na metade interior do Mar de Cananéia e na entrada do de Cubatão e Macoma sp. que ocorreu na baía do Trapandé e no Mar de Cubatão.

Dos gastrópodes devemos ressaltar a ocorrência de Littoridina australis var. nana que ocorreu apenas em estações realizadas na região de junção dos mares de Cananéia e de Cubatão (vêr Mınıussı, op. cit.) portanto, apenas nas regióes mais interiores dos mares de Cananéia e de Cubatão; fora daquela região, ocorreu apenas na estação 22. Turbonilla nivea ocorreu apenas na estação 7 (21 indivíduos/1/10 m²), situada na baía do Trapandé e em frente à barra de Cananéia.
Dos crustáceos, ressaltamos a ocorrência do tanaidaceo Kalliapseudes schubartii, nas estações 45, 46, 47 e 51, ou seja apenas no Mar de Cubatão. Sua maior densidade foi encontrada na estação 46 (45 indivíduos/1/10 $\mathrm{m}^{2}$ ). Upogebia brasiliensis ocorreu na estação 45, situada no Mar de Cubatão e Ogyrides occi. dentalis nas estações $22,32,52,53$ e 60 , portanto, em tôda a região estudada.

Os ofiuróides ocorreram apenas na região da baía do Trapandé e na estação 18. A espécie que ocorreu em maior número de estações foi Micropholis subtilis, mas sempre em densidade muito baixa, ou seja, de um indivíduo por estação.

Dos demais grupos bênticos, ressaltamos a ocorrência de Phoronis sp. nas estações 36, 45, 50 e 55 portanto, pràticamente, apenas no Mar de Cubatão e no de Itapitangui. A maior densidade desta espécie foi obtida na estação 50 (15 indivíduos/1/10 $\mathrm{m}^{2}$ ). Renilla reniformis ocorreu apenas na baía do Trapandé, salvo na estação 28, situada no Mar de Cananéia. Virgularia sp. ocorreu também apenas na baía do Tra. pandé, porém em apenas três estações, (estações 8, 9 e 58). A maior densidade de Renilla foi obtida na estação 8, (21 indivíduos/1/10 $\mathrm{m}^{2}$ ) e o de Virgularia na estação $58,\left(24\right.$ indivíduos/1/10 $\left.\mathrm{m}^{2}\right)$.

Os dados apresentados acima, mostram que as espécies comedoras de material em suspensão ocorreram, po rexcelência na região da baía do Trapandé, enquanto que espécies comedoras de detritos orgânicos dos sedimentos, ocorreram nos mares de Cananéia e de Cubatão. Na região de junção dêsses dois mares ocorreu predominantemente um microgastropode que podemos considerar como típico de regiốes estuarinas e lagunares com baixa salinidade, ou seja, a Littoridina australis var. nana.

Como se verifica da Tabela III, os grupos dominantes nas amostras obtidas na região da Ilha da Casca foram os poliquetas (estação 18, 19, 24) e os anfipodes (estações 20, 22). Os caranguejos foram dominantes apenas na estação 23. As estações 20 e 22 foram realizadas em fundo de pedra, enquanto que as estações 18, 19, 23, 24 em fundo de cascalho, areia e lôdo.

Verifica-se da Tabela IV, haver uma nítida diferença entre a fauna das estações 20 e 23 , realizadas em outro tipo de fundo. Em fundo de pedra ocorre uma rica epifauna, especialmente de hidróide Euden. drium carneum, esponjas, bem como numerosíssimas anfipodes, fauna esta, à primeira vista surpreendente para as condições hidrológicas e de sedimentação reinantes na região. Ai ocorrem também diversas algas principalmente Padina sp. e uma ceramiales e o ofiuróide Ophiothrix angulata. A fauna das estações 18 e 24, realizadas na entrada do canal mostra haver aí correntes, devido à presença de Owenia fusiformis, Chione subrostrata e Branchiostoma platae.

\section{ZONAS FAUNÍSTICAS}

Ainda que a amostragem seja preliminar, principalmente em função da complexidade hidrológica da 


\begin{tabular}{|c|c|}
\hline 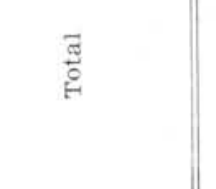 & MIIIIIIIIIIIIIII \\
\hline$\frac{1}{\partial}$ & 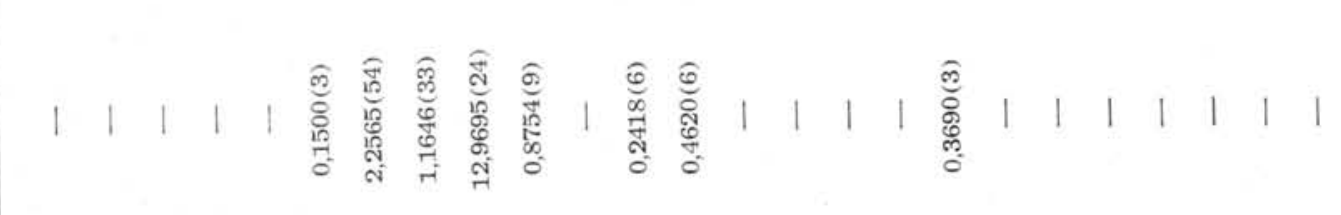 \\
\hline 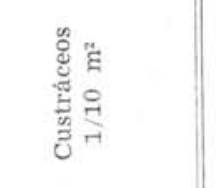 & 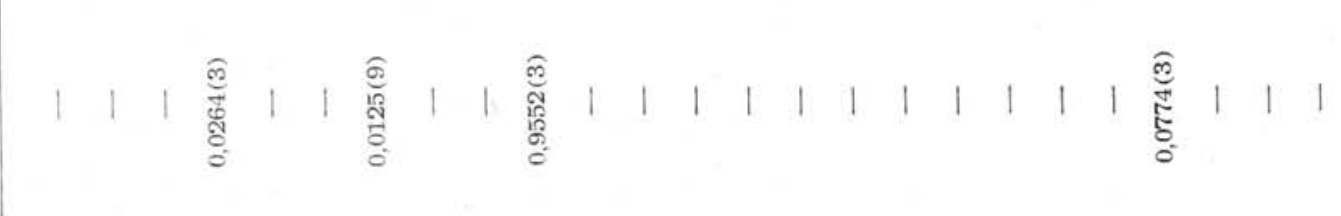 \\
\hline 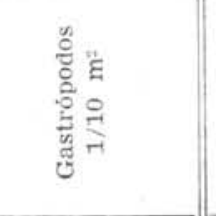 & 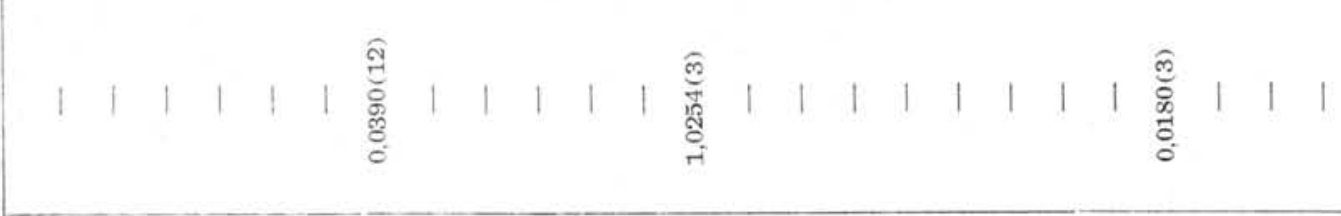 \\
\hline I & 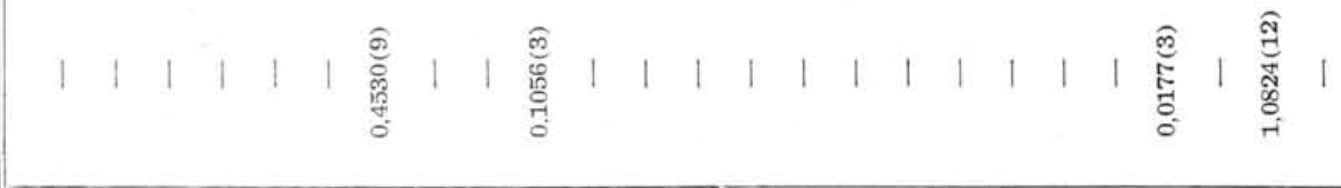 \\
\hline 焉 & IIUMUMIIIIIIII \\
\hline 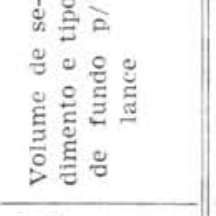 & $\begin{array}{ll}51 \\
01\end{array}$ \\
\hline 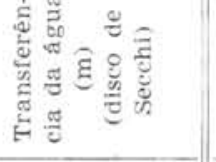 & 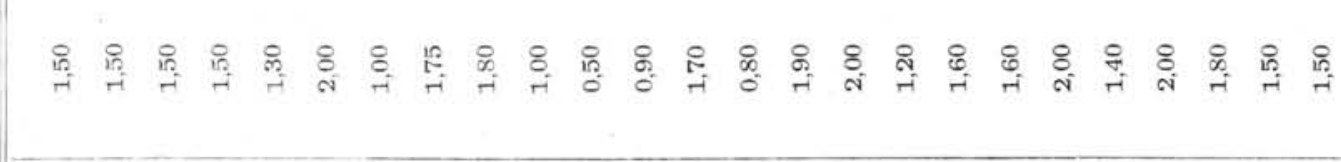 \\
\hline 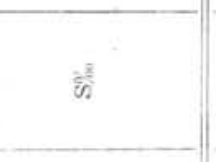 & 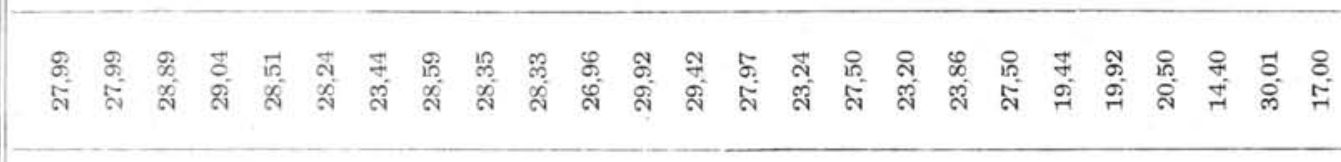 \\
\hline : & 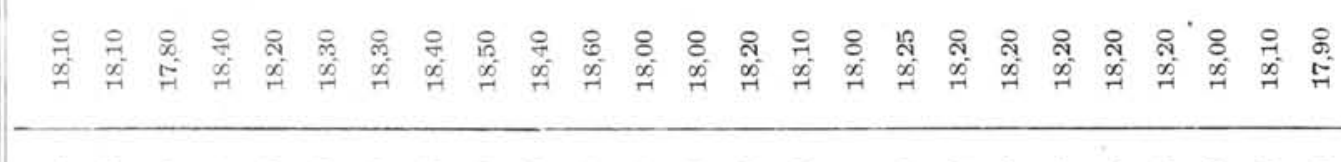 \\
\hline & 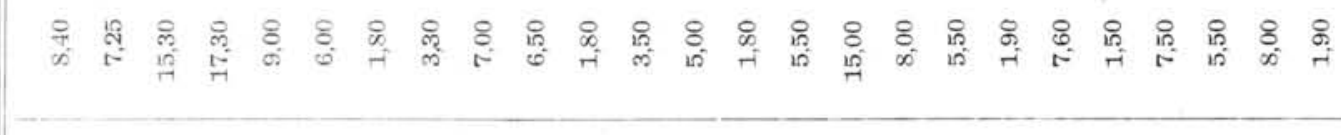 \\
\hline & \\
\hline
\end{tabular}




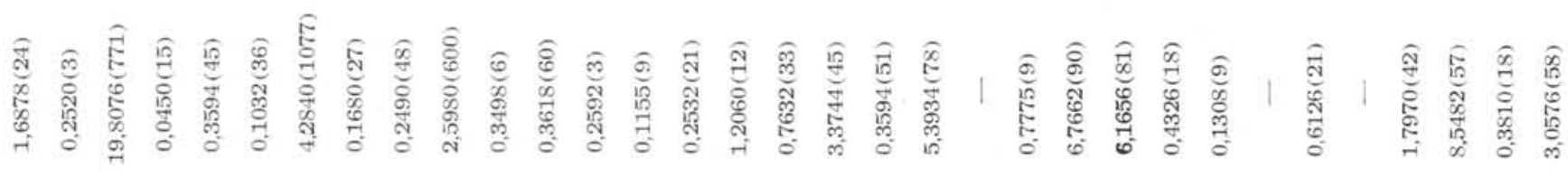

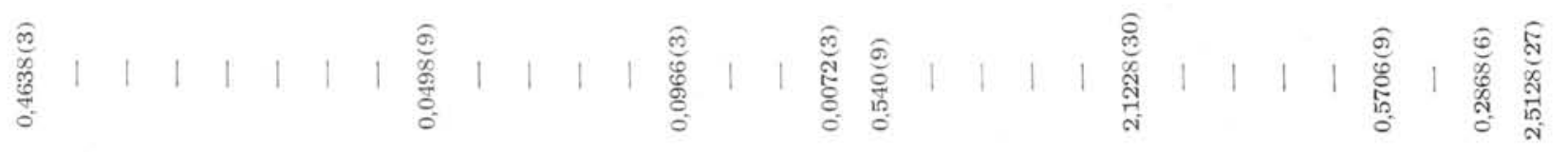

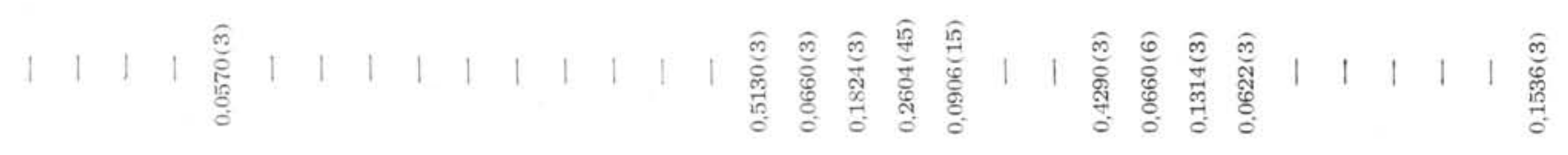

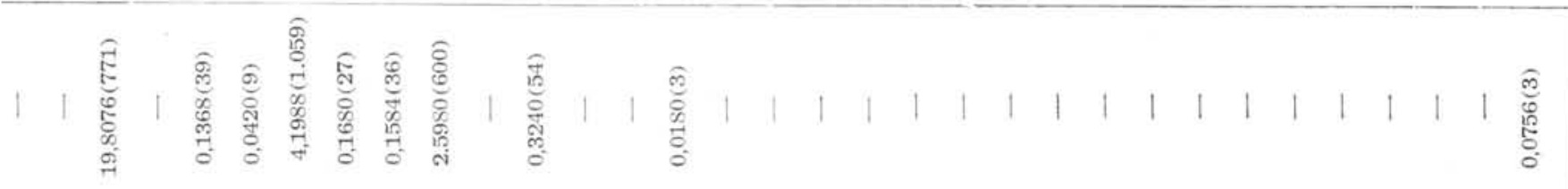

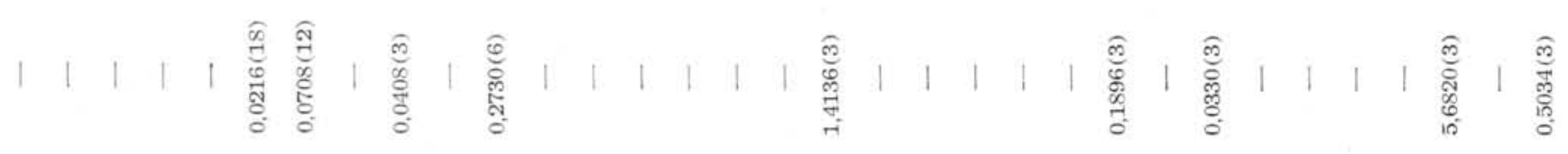

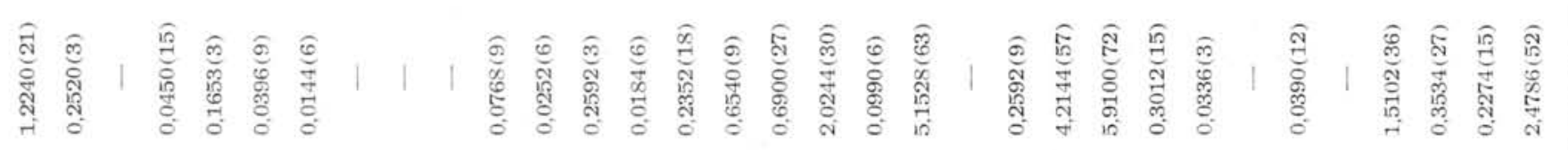

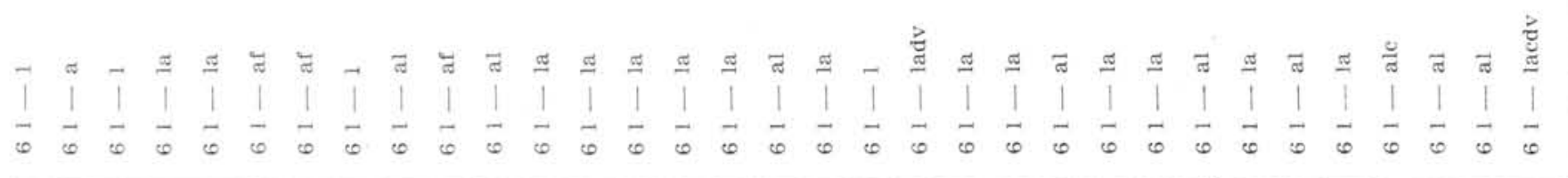

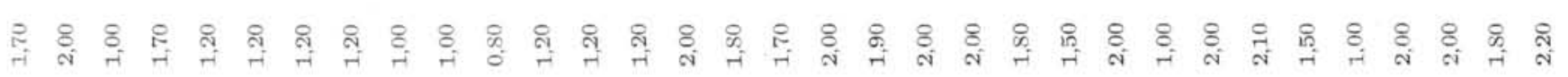

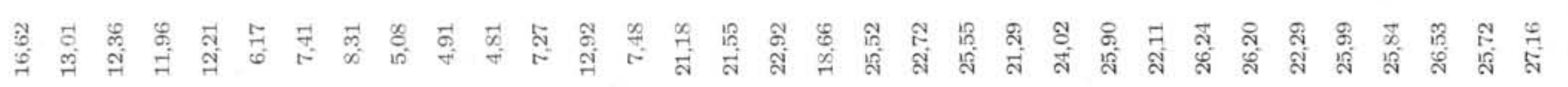

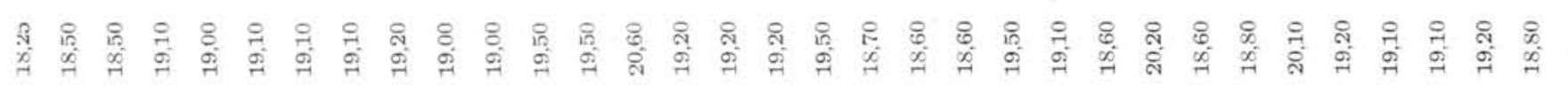

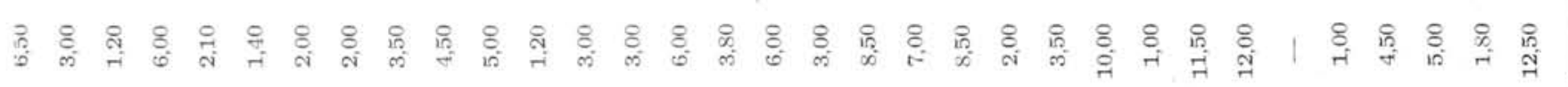
고용 


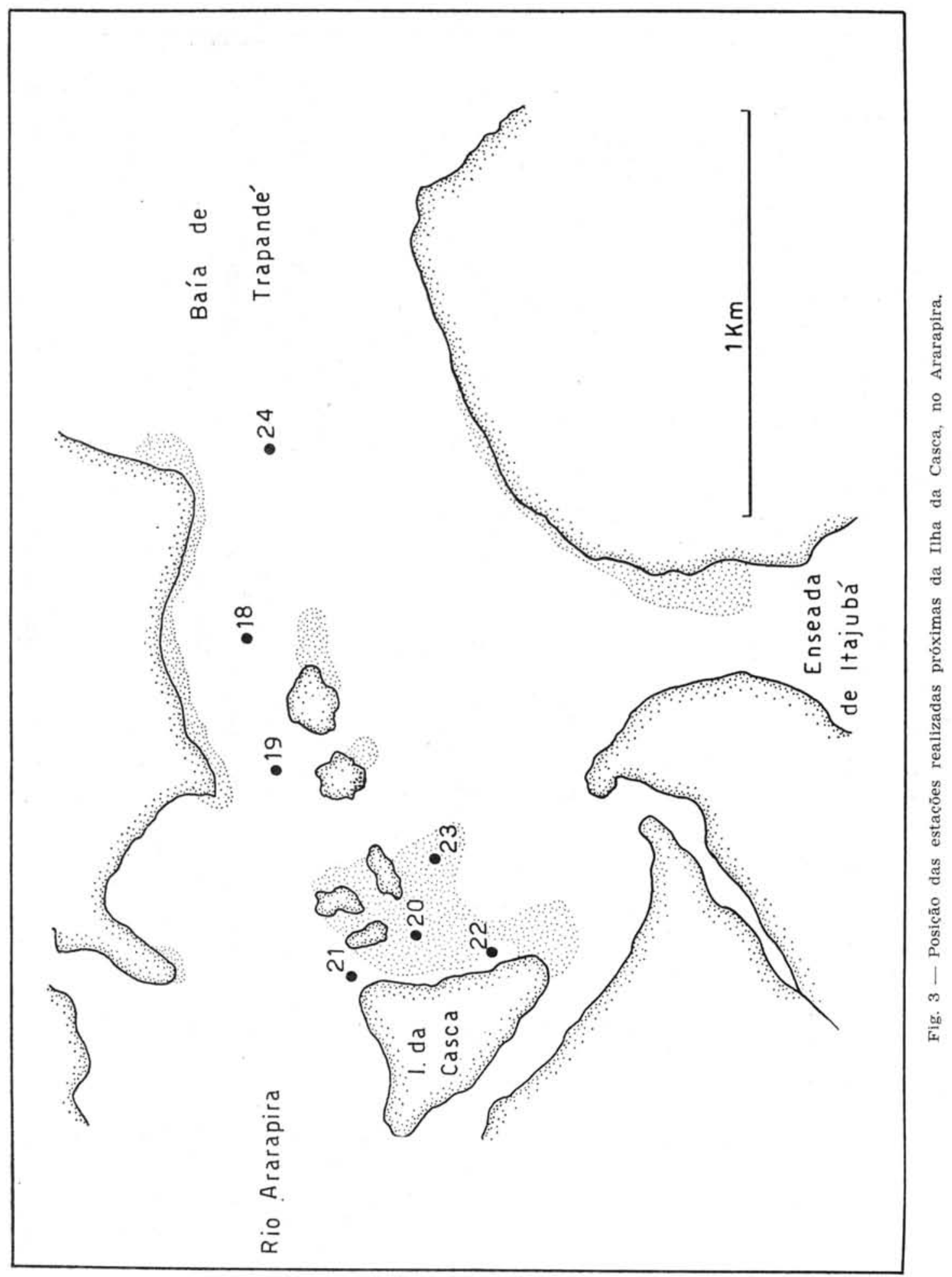


TABELA II - Lista das principais espécies coletadas na região infralitoral de fundos moles, do complexo estuarino-lagunar de Cananéia (SP)

ESPECIES

Polychaeta

Sigambra bassi

Ammotripane sp.

Amphinomidae

Armandia sp.

Clymene sp.

Clymenella sp.

Glycera sp.

Glycinde sp.

Goniada brunea

Laonice japonica

Loandalia americana

Magelona sp.

Nereis sp.

Ninoë sp.

Onuphis quadricuspis

Owenia fusiformis

Pilargis sp.

Poecilochetus australis

Prionospio pinnata

Prochoymene sp.

Telepsavus costarum

Mollusea

A mina mülleri

Arcopagia sp.

Lucina sp.

Macoma sp.

Tagellus sp.

Tellina alternata

Littoridina australis var. nana

Turbonilla nivea

Crustacea

Kalliapseudes schubartii

Ogyrides occidentalis

Pinnixa sp.

Pinnotheres sp.

Upogebia braziliensis

Antozoarios

Renilla reniformis

Virgularia sp.

Echinodermata

Amphipholis januarii

Diamphiodia riisei

Micropholis subtilis

Phoronoidea

Phoronis sp

$36(2), 45(1), 50(5), 55(3)$

$8(1), 60(2)$

$52(1)$

$60(7)$

$57(2)$

$58(3)$

$3(1), 58(1)$

$55(1)$

$57(5)$

$8(2), 9(2)$

$7(5), 8(2), 9(7)$

$10(2), 53(1), 57(2), 58(1)$

59 (1)

9 (2)

$10(1)$

7(1)

9(1)

$8(1), 10(1), 58(4)$

$10(3), 57(1), 60(1)$

$50(1)$

18(1)

$7(1), 13(1)$

$7(1)$

$58(1)$

$24(3), 45(1)$

7(4)

$45(1), 46(15), 47(5), 51(2)$

7 (1)

$10(1)$

45(1)

$8(2), 9(2), 58(8)$

$18(1)$

$12(2)$

$8(1), 9(1), 12(1), 18(1)$
$9(3), 10(9), 13(1), 47(9), 50(5), 51(5), 57(2), 60(14)$

$10(1), 24(1), 34(4), 53(1), 60(1)$

$7(1), 22(11), 33(6), 36(1), 38(2), 51(1)$

$22(1), 30(257), 32(13), 33(3), 34(353), 35(9), 36(12), 37(200), 39(18), 42(1)$

$22(11), 32(1), 52(1), 53(1), 60(1)$

$7(5), 8(7), 9(4), 10(3), 13(1), 28(1), 57(1), 58(1)$

$7(13)$

Branchiostoma platae 
região, pudemos verificar a existência de cinco zonas faunísticas, caracterizadas pelas seguintes espécies:

a) Zona de ofiuróides e de Renilla reniformis, Virgularia sp.: - Compreende a região das estações $7,8,9,12,18,57$ e 58 . Está situada na região da baía do Trapandé. Segundo Magliocca \& Kutver (1964) o sedimento dessa área apresenta menor conteúdo orgânico do que o das demais, correntes fortes com predominância das correntes de maré enchente sôbre as de maré vazante (KUTNER, 1962) e é a zona que sofre maior influência da água oceânica que entra pela barra de Cananéia (Teixeira, Tundisi \& Kutner, $1965)$.

$\left.b_{1}\right)$ - Zona de lamelibrânquios "deposit feeders" como Macoma sp. e Tellina alternata: - Compreende principalmente a região do Mar de Cananéia.

$\left.\mathrm{b}_{2}\right)$ - Zona do tanaidaceo Kalliapseudes schubartii e de Phoronis sp.: - Compreende a região do Mar de Cubatão, do Mar de Itapitangui até a foz do rio da Fôlha Larga. Pelos trabalhos de Kutner (1962) e de Magliocca \& Kutner (1964), verifica-se que os sedimentos do Mar de Cananéia e do Mar de Cubatão são diferentes, havendo maior conteúdo orgânico no de Cubatão. A isso provàvelmente estão relacionadas as diferenças faunísticas que observamos entre êsses dois "mares".

LOPES \& OKUDA (1968) verificaram haver uma relação inversa entre a abundância de artrópodes e a de moluscos. Segundo êsses autores os artrópodes predominam em estações aparentemente relacionadas com correntes, enquanto que nos locais onde predominaram moluscos as correntes foram insignificantes. No complexo estuarino-lagunar de Cananéia. como já mostramos, houve uma nítida separação de áreas entre o tanaidaceo Kalliapseudes schubartii e os lamelibrânquios Macoma sp. e Tellina alternata. Segundo Miniussı (1959) na região do Mar de Cubatão há um "tombo de água" o que não ocorre no Mar de Cananéia. Isso determina a ocorrência de condições ambientais diferentes, inclusive de correntes, nos dois "mares", como se verifica dos trabalhos de KutNer (1962), Magliocca \& Kutner (1964) e Kato (1966) e pode ser a responsável pelas diferenças faunísticas observadas.

TABELA III - Número de individuos dos principais grupos bênticos em relação a $1 / 10 \mathrm{~m}^{2}$

Est. 18 $5,40 \mathrm{~m}$ de profundidade.

Fundo de areia, lôdo e conchas.

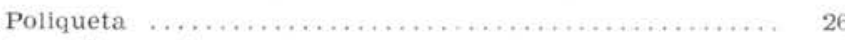

Lamelibranquia

Gastropoda

Decapoda

Amphipoda

Outros ....

Est. 19

$6 \mathrm{~m}$ de profund.uade

Poliqueta

Outros

Fundo de arela fina e lôdo.

Est, 20

$6 \mathrm{~m}$ de profundidade

Fundo de pedras.

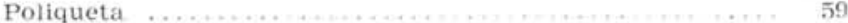

Lamelibranquia ......................................... 114

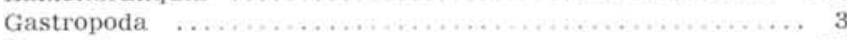

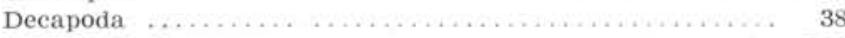

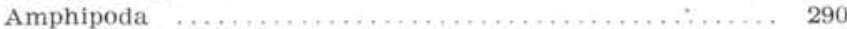

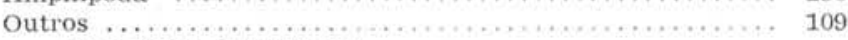

(Hydrozoa, Nematoda)

Est. 21

$6 \mathrm{~m}$ de profundidade.

Fundo de pedras.

Est. 22

$8 \mathrm{~m}$ de profundidade.

Fundo de pedras.

Decapoda

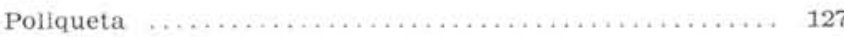

Lamelibranquis. ............................. 47

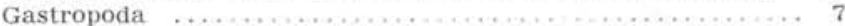

Decapoda ....................................... ${ }_{2}^{2}$

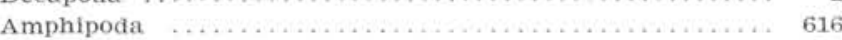

Outros ................ 75

(Nemaioda, Isopoda Nemertinea, Hydrozoa, Porifera)

Est. 23

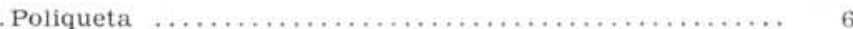

$8 \mathrm{~m}$ de profunidade

Fundo de pedras.

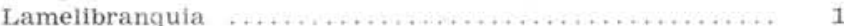

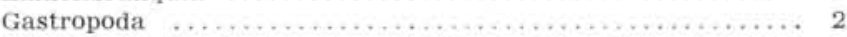

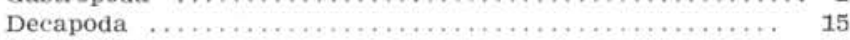

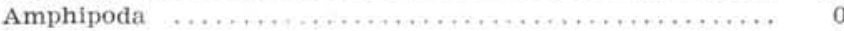

Outros ...................................... 9

Est. 24

Poliqueta ....................................... 13

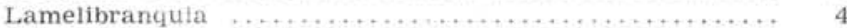

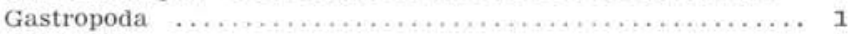

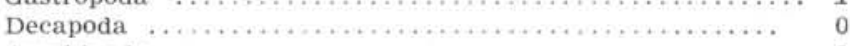

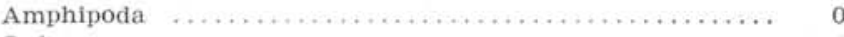

Outros ................................... 4 
TABELA IV - Principais espécies encontradas na região da Ilha da Casca

\begin{tabular}{|c|c|c|c|c|c|c|c|}
\hline Poliquetas & 18 & 19 & 20 & 21 & 22 & 23 & 24 \\
\hline 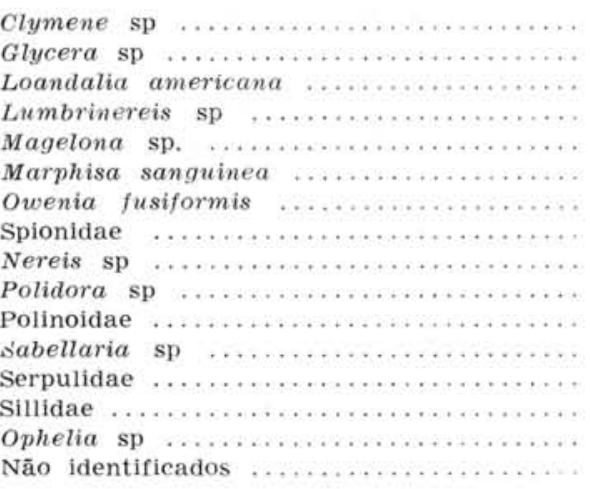 & $\begin{array}{r}1 \\
2 \\
12 \\
1 \\
1 \\
2 \\
4 \\
1\end{array}$ & $\begin{array}{r}2 \\
1 \\
10\end{array}$ & $\begin{array}{r}1 \\
5 \\
24 \\
2 \\
1 \\
25\end{array}$ & & $\begin{array}{r}5 \\
\\
2 \\
59 \\
2 \\
48 \\
11\end{array}$ & . & $\begin{array}{l}1 \\
3\end{array}$ \\
\hline
\end{tabular}

Diversos

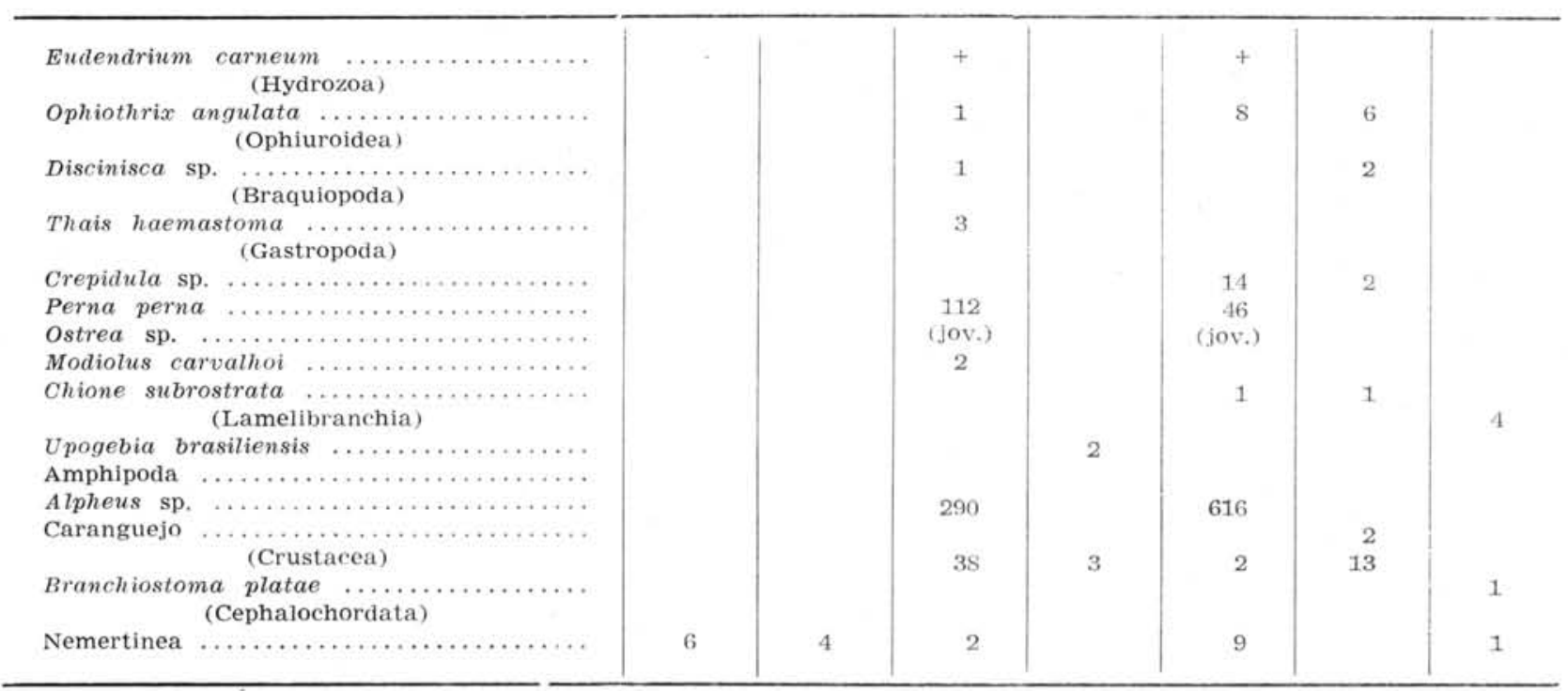

$+=$ numerosas colonias

c) Zona do Hydrobiideo Littoridina australis var. nana - Abrange a região norte da Ilha de Cananéia, da Ilha das Garças até a foz do Rio Baguassu. Apresenta salinidade baixa de $\left(4,91\right.$ a $\left.12,36_{\% 0}^{\circ}\right)$, sedimento com muita matéria orgânıca. Esta zona apresenta, de um modo geral, menos matéria orgânica em suspensão (Teixeira, Tunisi \& Kutver, 1965). baixa transferência e o mais elevado volume de fitoplâncton (células/litro). Segundo MARcus E. \& E. (1963) esta espécie é comedora de epistrato. A elevada densidade desta espécie na região norte da llha de Cananéia é provàvelmente devida à ausência de com. petidores e predadores nessa região (vêr SANDERs, 1968) e às condições de "pessimus" ai existentes para êsses elementos da fauna bêntica.

d) Zona de fundos duros da região da llha dit Casca, com numerosos Eudendrium carneum, Ophiothrix angulata, numerosíssimos anfipodes, etc,
Os resultados obtidos por Machado (1952), e a proximidade da região da Itha da Casca, da barra de Cananéia, explicam largamente, a riqueza da epifauna do fundo duro infralitoral, dessa região. Sendo uma região de junção de águas (МАснADo, op. cil.), aí devem ocorrer condiçôes muito favoráveis à exislência de "suspension feeders" como foi demonslrado para outras regiōes (UrSIN, 1960; PETERSEN, 1914; Steemann-Nielsen, 1940). A presença de abundante epifauna na região da Ilha da Casca indica, a presença de um suporte estável çue não é coberto pelo lôdo e sugere e existência de um fundo em equilíbrio, onde as correntes são suficientemente fortes para evitar uma deposição intensa (vêr Bort.ır, 1964).

Graças a êsses mecanismos, a região da llha da Casca seria uma das poucas se não a única, do complexo estuarino-lagunar de Cananéia a apresentar uma rica associação infralitoral de fundo duro. 


\section{OBSERVAÇÕES GERAIS}

De tôda a região de fundos moles do complexo estuarino-lagunar de Cananéia, a da baía do Trapandé, especialmente as estações 9,10 e 58, é a que parece possuir fauna mais variada. Nessa baía foi também encontrada elevada biomassa bêntica (em pêso alcoólico). A razão dessa riqueza pode ser compreendida dos trabalhos de Teixeira, Tundisi \& Kutner (1965) e de Kato (1966) que mostraram haver uma menor variação da salinidade da água de fundo na área mais próxima da barra, ou seja, na baía do Trapandé, do que nas demais áreas da região. Isso deve ter sem dúvida, influência marcante na distribuição da fauna bêntica.

Segundo, os resultados de Magliocca \& Kutner (1964) verifica-se também que nessa baía encontra-se a região na qual as condições sedimentológicas (matéria orgânica, granulometria) são mais favoráveis à fauna bêntica de origem oceânica. Isso se deve por ela estar mais sob influência direta da água vinda do oceano do que as demais regióes e pela ausência de desembocaduras de grandes rios nessa região e, portanto, de fundos ricos em material floculado pela mistura de água do mar com a oriunda de rios e "marigots". Êsse material pode formar um depósito não consolidado de vários centímetros de espessura sôbre o sedimento, criando assim condições muito desfavoráveis à sobrevivência da macrofauna. Essa é, provàvelmente, a razão da não existência de animais nas amostras das estações 11, 14, 17, 20, 48,54 e 56. A ausência de animais nas estações 2 e 5 se deve, provàvelmente, por êsses locais estarem sob ação das fortes correntes que ocorrem na região da barra de Cananéia.

As estações 8 a $11 ; 12$ a 14 e 56 a 59 realizadas na baía do Trapandé, as estações 12 a $14 ; 15$ a 17 ; 19 a $21 ; 23$ a $25 ; 27$ a $29 ; 30$ a $32 ; 33$ a 35 e 36 a 38 realizadas no Mar de Cananéia e as estações 39 a $41 ; 43$ a $45 ; 47$ a 49 e 50 a 52 realizadas no Mar de Cubatão mostraram os seguintes padrões de distribuição nos cortes representados por cada um dêsses conjuntos de estações.

a) Na baía do Trapandé, as estações realizadas próximas à Ilha de Cananéia (Est. 14, 11 e 56) não apresentaram fauna bêntica, provàvelmente, devido ao lôdo fino, floculado, existente no fundo. A estação 7 , está sob ação direta da água oceânica vin. da da barra ae Cananéia e por isso apresentou fauna bêntica.

b) A região entre 3 a 5 metros de profundidade mais próxima da Ilha do Cardoso (estações 9, 13 e 58) é a mais rica em fauna bêntica da baía do Trapandé. As estações 8, 12 e 59, realizadas próximo da Ilha do Cardoso, ao contrário das realizadas próximo da Ilha de Cananéia, revelaram fauna bêntica, pois o sedimento foi menos fino do que o dessa região e por estar mais sujeito à água oceânica vinda da barra de Cananéia.

c) Nos cortes representados pelas estações 15 a $17 ; 23$ a $25 ; 27$ a $29 ; 33$ a 35 e 36 a 38 , realiza- dos no Mar de Cananéia, houve mais fauna bêntica no meio do mar do que nos lados; já nos cortes representados pelas estações 19 a 21 e 30 a 32 , houve mais fauna no lado próximo da Ilha Comprida (estações 19 e 30). É interessante verificar que êsses dois cortes foram realizados em regiōes de concavidade da Ilha Comprida. É possível que haja relação entre a circulação da água e essa anomalia de dis. tribuição da fauna bêntica.

d) No mar de Cubatão a distribuição da fauna foi bem diferente da do Mar de Cananéia. As regiões próximas à Ilha de Cananéia (est. 39, 47 e 50) têm fauna mais abundante; as mais distanciadas da Ilha (estações 44 e 48) e as mais próximas do continente (Estações 41 e 52) são mais pobres.

\section{DISCUSSÃO}

Como observou muito bem DAY (1967), em um estuário cada um dos parâmetros ambientais, especialmente as correntes, a salinidade, a temperatura da água e a natureza do fundo têm efeitos particulares sôbre a fauna estuarina. Porém, como um estuario é um ecosistema unificado, todos os fatôres ambientais atuam uns sôbre os outros para determinar a natureza e distribuição da fauna (DAY, op. cit., p. 397).

Nos componentes da fauna bêntica infralitoral de fundos moles, do complexo estuarino-lagunar de Cananéia (SP), podemos reconhecer dois grupos: o primeiro constituído por espécies oriundas do mar que penetraram e se estabeleceram na região. Inclui, entre outras, Tellina alternata, Turbonilla nivea, Renilla reniformis, Virgularia sp. e os ofiuróides Amphipholis januarii, Diamphiodia riisei e Micro. pholis subtilis. O segundo, formado por espécies estuarinas, como Littoridina australis var. nana é restrito à região norte da Ilha de Cananéia.

Segundo CARriker (1967) a repopulação dos "Taxa" bênticos nos estuários ocorre de estação para estação, principalmente por reforço vindo do mar e, em menor extensão, do próprio estuário. Os detalhes de como o recrutamento ocorre ao nível das espécies individuais, permanecem obscuros.

$\mathrm{Na}$ presente região, o estudo da fauna bêntica infralitoral de fundos moles é complexo pois não se trata de um estuário, mas de um complexo estuarino-lagunar (Teixeira, 1969). Talvez devido a isso hajam oscilações na composição específica dessa fauna, maiores do que em um estuário típico.

Muitos estudos têm mostrado que o número de espécies marinhas é mais abundante na bôca dos estuários, decresce, e, finalmente, desaparece nas partes internas do mesmo (CARRIKER, op. cit., p. 463). Isso ocorre na região presentemente estudada, pois a região com maior variedade de fauna foi a baía do Trapandé. Daí, tanto pelo mar de Cananéia, como de Cubatão, em direção à região norte da Ilha de Cananéia há uma redução da fauna bêntica (ver Tabelas I e II). 
As diferenças faunísticas observadas entre o mar de Cananéia e o de Cubatão são, provàvelmente, devido a que na região do Mar de Cubatão (Fig. 1) abrem-se rios bem maiores do que no de Cananéia, que fica entre duas ilhas muito longas, mas relativamente estreitas e com "marigots" menores do que os rios que se abrem na região continental do Mar de Cubatão.

\section{AGRADECIMENTOS}

Ao Dr. Edmundo F. Nonato, pela classificação dos poliquetas e valiosas sugestóes, à Dra. Marta Vannucci, pela classificação do hidróide, ao Dr. Plínio Soares Moreira pela classificação dos tanaidaceos, ao Dr. Clóvis Teixeira por diversas observaçôes e à Lic. Licia Penna, pela classificação dos lamelibrânquios, nossos sinceros agradecimentos.

\section{SUMMARY}

The Cananeia region in the south of São Paulo State, is a estuarine-lagunar complex. In this region we realesed 60 benthic stations with a Foerst "Petersen grab" of 1/10 sq.m. We had studied the density and alcoholic weight of the benthic groups and species. The polychaets was the dominant group in number of species and of individuals. The commonest species was Loandalia americana, Laonice japonica, Clymene sp., Clymenella sp.. The highest density of polychaets was obtained in station 51, 10 meters deepthty, temperature of $18.60{ }^{\circ} \mathrm{C}$, salinity of $25.90 \%$, water transparency (Secchy disc) of 2 meters, bottom of muddy, sand and pebles. In this station we found 72 individuals $/ \mathrm{sq} . \mathrm{m}$. and a alcoholic weight of $5.9100 \mathrm{grm}$. The lamelibrancs was not dominant in neither one station. Tellina alternata and Macoma sp. occurred only in Trapandé bay and Cubatão sea. Littoridina australis var, nana occurs in high density in the north region of Cananéia island. In this region it is the dominant benthic species. Kalliapseudes schubartii occurs only in the Cubatão sea. Renilla reniformis and Virgularia sp. occurs specially in the Trapandé bay.

It was possible to recognize five faunistics zones in the soft bottoms of the complex. Zone of Renillu reniformis and Virgularia sp.. The sediment of this zone have a low organic fraction and the zone is on direct influence of the ocean waters. Zone of Macoma sp. and Tellina alternata in the Cananéia sea. Zone of the tanaidaceous Kalliapseudes schubartii and Phoronis sp. principally in the Cubatão sea. In this sea there are the highest organic fraction of the sediment. Zone of Littoridina australis var. nana in the northern region of the Cananéia island. In this zone, we found a low transparency of the water, salinity of 4.91 to $12.36 \%$ and a high organic fraction in the sediment. Zone of hard bottoms with hydroids and ophiuroids.

The Trapande bay, presents the richest muddy infralittoral benthic fauna of the complex. In this bay is found the highest oxygen content and water highest salinity of the complex. Near of the mouth of the rivers and of the "marigots" and in many places of the complex, we found a floculated softy sediment in the bottom. In these places we d'ont found animals or they are very much rare (stations 11, 14, $17,20,48,54$ and 56). In two stations near of the Cananéia bar, in result of strong currents we d'ont found animals (St. 2, 5).

Inside of the estuarine-lagunar complex of Cananéia (SP) characterized by the presence of muddy and sand-muddy bottoms with a very much poor epifauna we found in the Casqueiro island a infralittoral stone bottom, with a rich epifauna, specially of the hydroids Eudendrium carneum Clarke, the Ophiothrix angulata Say, amphipods, etc. This occurs because this area is situated in a region of function of the waters of the Trapandé bay and that of the Ararapira river, what permits the existence of a stone bottom not covered by the muddy. This situation permits the occurrence of that epifauna.

\section{BIBLIOGRAFIA}

ALLEE, W. C., PARK, O., EMERSON, A. E., PARK, T. \& SCHMIDT, K. P.

1963. Principles of animal ecology. Philadelphia, Saunders, 837 p.

BOILLOT, G.

1964. Géologie de Manche Occidentale. Annls Inst. océanogr., Monaco, vol. 42, 220 p.

CARRIKER, M. R.

1967. Ecology of estuarine benthic invertebrates, a perspective. In: Lauff, G. H., ed. - Estuaries. Washington, Am. Ass. Adv. Sci., Publ. 83, p. 442-587.

DAY, J. H.

1967. The biology of Knysna estuary, South Africa. In: Lauff, G. H., ed. - Estuaries. Washington, Am. Ass. Adv. Sci., Publ. 83, p. 397-407.

GERLACH, $\mathrm{S}$.

1958. Die mangroveregion Tropischer Küsten als lebensraum. Z. Morph. Ökol. Tiere, vol. 46 , p. $636-730$

1963. Ökologische Bedeutung der Küste als Grenzraum zwischem Land und Meer. Naturw. Rdsch., Stuttg., vol. 16, n.॰ 6, p. 219-227.

HESSE, R., ALLEE, W. C. \& SCHMIDT, K. P

1937. Ecological animal geography. New York, John Willey, $597 \mathrm{p}$.

KATO, K

1966 Chemical investigations on the hydrographycal system of Cananéia lagoon. Bolm Inst. oceanogr. S. Paulo, vol. 15, n. 1 , p. $1-12$.

KUTNER, A

1962 Granulometria dos sedimentos de fundo da região de Cananéia, S.P. Bolm Soc. bras. Geol., vol. 11, n. 2 , p. 41-54

LOPES, M. A. \& OKUDA, T.

1968 Algunas observaciones sobre caracteristicas físico-químicas de los sedimentos y distribución de la fauna macro bentónica de la Laguna Grande del Obispo. Boln Inst, oceanogr. Univ. Orient, vol. 7, n. ${ }^{\circ}$ 1, p. 107-128.

MACHADO, L. B.

1952. Pesquisas físicas e químicas do sistema hidrográfico da região lagunar de Cananéia. II Águas de junção. Estudos iniciais das águas comuns da região da Ilha da Casca. Nota preliminar sôbre as águas, na juncão do Canal de Ararapira e Baia de Trapandé. Bolm Inst. oceanogr. S. Paulo, vol. 3 , n. ${ }^{\circ} 1 / 2$, p. $55-75,2$ mapas. 
MAGLIOCCA, A. \& KUTNER, A. S.

1964. Conteúdo orgânico dos sedimentos de fundo de Cananéia, São Paulo. Contrcōes Inst, oceanogr, S Paulo, ser. Ocean. fis., n. ${ }^{\circ}$ 7, 14 p.

MARCUS, E. \& MARCUS, E.

1963 Mesogastropoden von der Küste São Paulo. Abh. math. naturw. K1. Akad. Wiss. Mainz, n. ${ }^{\circ} 1,105$ p.

MINIUSSI, I. C

1959. Propagação da onda de maré em tôrno da Ilha de Cananéia. Contrções Inst. oceanogr. S. Paulo, sér. Ocean. fis., n." 2,5 p. 3 figs.

PETERSEN, C. G. J.

1914. Valuation of the sea II. The animal communities of the sea bottom and their importance for marine zoogeography. Rep. Dan. biol. Stn, n. ${ }^{\circ} 21$, p. 1-44, 1-68.

SANDERS, H. L.

1968. Marine benthic diversity: a comparative study. Am. Nat., vol. 102 , n.o 925, p. 243-282.

STEEMANN-NIELSEN, E.

1940. Die produktionsbedingungen des phytoplanktons im Übergangsgebiet zwischen der Nord und Ostsee. Meddr Danm. Fisk.-og Havunders., vol, 3, n. ${ }^{\circ} 4,55$ p.

TEIXEIRA, C.

1969 Estudo sôbre algumas características do fitoplâncton da regiảo de Cananéia e o seu potencial fotossintético. Tese de doutoramento apresentada à Universidade de São Paulo.
TEIXEIRA, C. \& KUTNER, A.

1961. Contribuição para o conhecimento das diatomáceas da regiã̃o de Cananéia Bolm Inst. oceanogr. S Paulo, vol. 11, n. ${ }^{\circ} 3$, p. $41-74$.

TEIXEIRA, C., TUNDISI, J. \& KUTNER, M. B.

1965. Plankton studies in a mangrove environment. II. The standing stock and some ecological factors. Bolm Inst. oceanogr. S Paulo, vol. 14, p. 13-42.

THORSON, G.

1957 Bottom communities (sublittoral or shallow shelf). In: Hedgpeth, J. W., ed. Treatise on marine ecology an paleoecology. Washington, Geol. Soc. Am., Memoir 67, vol. 1, p. 461-534.

TOMMASI, L. R.

1967. Observações preliminares sôbre a fauna bêntica de sedimentos moles da baia de Santos e regiões vizinhas. Bolm Inst. oceanogr. S Paulo, vol. 16, p. 43-66.

TUNDISI, J. \& TUNDISI, T. M.

1968. Plankton studies in a mangrove environment. V. Salinity tolerances of some planktonic crustaceans. Bolm Inst. oceanogr. S Paulo, vol. 17, p. 57-65.

URSIN, E.

1960. A quantitative investigation of the echinoderm fauna of the Central North Sea. Meddr Danm. Fisk.-og Havunders., n.s., vol. 2, n. ${ }^{\circ} 24$, p. 1-204. 